\title{
Alfabetización en salud en contexto penitenciario: programa de educación por \\ pares
}

\section{Health literacy in prison setting: A peer education program}

\author{
Gloria Jólluskin*, Isabel Silva*, Andreia de Castro-Rodrigues \\ *FP.B2S, UFP, **UM, ULP
}

\begin{abstract}
Resumen
El presente trabajo describe la planificación de un programa de educación para la salud en contexto penitenciario, utilizó una metodología comunitaria y de educación por pares. El programa tenía como objetivo aumentar el bien estar físico y emocional de los internos y dotarlos de estrategias para poder enfrentar los retos de la reclusión. Identificamos las necesidades de intervención a través de entrevistas a informadores clave y a los internos. Posteriormente, diseñamos e implementamos la intervención, desarrollando ocho sesiones que evaluamos con un cuestionario. Fueron elaborados carteles divulgativos de mensajes de salud para transferir conocimientos a los restantes internos.

Palabras clave: educación correccional, salud, educación para la salud mental, grupo de iguales.
\end{abstract}

\begin{abstract}
This paper describes a health promotion program in prison setting, using a communitarian and peer education methodology. The principal objective was to increase physical and emotional well-being of prisoners, as well as to provide them with useful strategies to face the challenges posed by detention. Intervention needs were identified through interviews with key informants and inmates. Subsequently, the intervention was planned and implemented, incorporating the interests and motivations of both - professionals and inmates. Eight sessions were conducted and evaluated through a questionnaire. Posters with health messages were created by participants to transfer knowledge to the other inmates.

Keywords: correctional education, health, mental health education, peer group.
\end{abstract}

Aunque los programas de promoción de la salud y la alfabetización en salud son intervenciones relativamente frecuentes, apenas unos pocos se han dirigido a personas en situación de reclusión, si bien este grupo presenta importantes necesidades de salud (Enggist, Møller, Galea, \& Udesen, 2014; Santora, Espnes, \& Lillefjell, 2014). Según la Organización Mundial de la Salud (OMS), la población carcelaria presenta niveles de salud más bajos que la población general, no siendo extraño que muchos internos entren en contacto por primera vez con los servicios de salud durante su condena (Møller, Stöver, Jürgens, Gathere, \& Nikogosian, 2007). Los centros penitenciarios suelen estar diseñados y gestionados bajo la lógica de la vigilancia y el control, imponiéndose normas de conducta con rigidez y dureza, lo que no favorece las condiciones necesarias para una promoción de la salud, dadas las consecuencias psicológicas que esta imposición de normas tiene para los internos (Watson, Stimpson, \& Hostick, 2004). Pero los riesgos para la salud asociados a las condiciones de los centros penitenciarios afectan también a la población en general, a través de los visitantes y el personal penitenciario (Comfort \& Grinstead, 2004). Así, no es de extrañar que la salud de los presos haya sido reconocida por la OMS como un problema de salud pública, defendiéndose la promoción de la salud en contexto penitenciario como una prioridad social (Møller et al., 2007), a pesar de la limitación de recursos, a través de un enfoque comunitario, y sondando las opiniones de internos y profesionales para identificar necesidades y aplicar las estrategias más eficaces (WHO, 1995). Esta promoción de la salud debería contemplar aspectos como la capacitación y el empoderamiento; componentes físicos y mentales de la salud; la necesidad de extender estos cuidados a la comunidad; y, finalmente, la aplicación de un enfoque multidisciplinario y holístico (WHO, 1995).

Promover la resiliencia en contexto penitenciario parece fundamental, no sólo para conseguir un mejor estado de salud de los internos, sus familias y la comunidad en general, contribuyendo a la reducción de las desigualdades en salud, sino también para aumentar la probabilidad de que el reingreso en sociedad se produzca con éxito (Enggist et al., 2014). De hecho, se ha relacionado la participación en actividades prosociales durante el encarcelamiento con la prevención del desajuste y las infracciones subsiguientes, mejorando la conducta que rompe las reglas y reforzando el comportamiento convencional (Collica-Cox, 2014; Enggist et al., 2014).

Considerando todo lo anterior, desarrollamos el programa "Salud en Cadena", con el objetivo de desarrollar la alfabetización en salud entre los internos, responsabilizándolos e involucrándolos en la toma de decisiones sobre la salud, al mismo tiempo que se intentaba promover competencias básicas de comunicación en salud, así como desarrollar sus potencialidades en este ámbito. Para ello, se pretendió 
formar un pequeño grupo de reclusos que divulgasen mensajes de salud entre la población penitenciaria, siguiendo una lógica de educación por pares. La utilización de este tipo metodología influenció el nombre del programa, por referencia a la palabra "cadeia" ("cadena" en portugués), un nombre utilizado en lenguaje común para designar a la prisión, al mismo tiempo que nos referíamos a la "reacción en cadena" que pretendíamos provocar a través de la formación de un pequeño grupo de individuos. El juego de palabras se pierde en la traducción. El equipo responsable por la planificación e implementación del programa estaba compuesto por profesionales externos al centro penitenciario.

\section{$1^{a}$ Fase. La evaluación de necesidades}

Después de obtener autorización de la Direção Geral de Reinserção e Serviços Prisionais y de la Dirección del Centro Penitenciario para implementar el programa, procedimos a la evaluación de necesidades. Esta fase incluyó entrevistas en profundidad con dos grupos de informantes clave: 10 profesionales y el grupo de internos que participaron en el programa. Solicitamos la colaboración de los profesionales que trabajan directamente con los internos para realizar entrevistas semiestructuradas que nos permitiesen identificar las principales necesidades de salud de la población penitenciaria, centrándonos particularmente en los problemas de salud más comunes, los recursos sanitarios existentes, así como la calidad de los mismos y el uso que los internos daban a esos recursos. Finalizamos la entrevista con preguntas centradas en la satisfacción de las necesidades de salud identificadas. Los profesionales entrevistados desenvolvían funciones en áreas como Enfermería (5 participantes), Psiquiatría (1), Educación (1), Terapia Ocupacional (1), Mediación (1), Psicología (1) y Magisterio (1).

Paralelamente, solicitamos a la Dirección ayuda en la selección de los internos que recibirían la intervención para ser agentes de salud a partir de las siguientes características: capacidad de liderazgo; abertura, respeto e flexibilidad en relación a los otros y otras realidades; capacidad para identificar e desenvolver competencias e habilidades en los otros; permanencia en el centro penitenciario de por lo menos un año; estar comprometido con temas de salud; competencias de trabajo en equipa; interés en participar no proyecto. Posteriormente, la Dirección nos proporcionó una lista de 11 internos seleccionados para formar el grupo. Durante el primer contacto que tuvimos con ellos, tuvimos la oportunidad de recoger las necesidades de intervención a partir de sus testimonios, realizando una entrevista grupal. Iniciamos esa actividad con una conversación informal sobre la salud y la alfabetización en salud, para seguidamente iniciar una lluvia de ideas, solicitando a los participantes que señalasen las temáticas que para ellos sería interesante tratar durante el programa. Esto nos permitió obtener una respuesta espontánea sobre las expectativas que ellos tenían en relación al programa, al mismo tiempo que les proporcionó un espacio en el que se les permitió expresar sus opiniones sobre los contenidos a incluir. A continuación, se presentó a los internos una serie de cartones con otras propuestas de temas que habíamos previamente identificado en otros programas de alfabetización en salud en contexto penitenciario (Equip Vincles Salut, 2011; Scientific Institute of the Medical Association of German Doctors, 2013), solicitándoles que se pronunciasen sobre la relevancia de tratar esos aspectos durante las sesiones de grupo. Pretendíamos así incluir temáticas que, no habiendo sido identificadas de forma espontánea, fuesen consideradas relevantes, así como que los internos eliminasen aquellas temáticas que no se correspondiesen con las necesidades sentidas por el grupo.

\section{$2^{a}$ fase. La planificación del programa}

Como fue anteriormente mencionado, el contenido de las sesiones del programa se basó en la revisión de la literatura, incluyendo algunas experiencias previas desarrolladas en contexto penitenciario (Equip Vincles Salut, 2011; Scientific Institute of the Medical Association of German Doctors, 2013), así como en la evaluación de las necesidades realizada a partir de las entrevistas con los profesionales y la entrevista grupal desarrollada con los internos durante el primer contacto (Sesión 0 ), en la cual se procedió a la validación de los contenidos del programa.

Durante la primera sesión, el equipo presentó a los participantes una propuesta de intervención consistente en 8 sesiones de trabajo semanales, que fue aprobada por el grupo. En la tabla 1, presentamos un breve resumen de los contenidos incluidos en el programa. Igualmente, se definieron las normas de funcionamiento del grupo, así como los principios clave y técnicas didácticas a utilizar. Con el objetivo de promover la internalización de los contenidos, fueron propuestas técnicas interactivas y participativas (García, Gutiérrez, \& Morante, 2004), que promoviesen el intercambio de opiniones, la adquisición de actitudes que funcionasen como factor de protección, así como las capacidades de modificar la conducta a través de actividades lúdicas y significativas basadas en la experiencia (Bustamante et al., 2013).

Para promover las estrategias de aprendizaje, intentamos transmitir apenas datos reales, creíbles, significativos y actualizados, e incluir momentos participativos, antes y después de los momentos expositivos. Por otro lado, intentamos siempre promover y reforzar la participación de los reclusos, incluyendo sus contribuciones a la sesión, con el objetivo de fomentar una experiencia de aprendizaje significativa y funcional mediante el establecimiento de vínculos entre los nuevos contenidos a aprender y el conocimiento previo de los participantes (Coll \& Solé, 1989).

Después de la presentación a los internos de los contenidos del programa y de obtener una validación de los mismos, solicitamos a estos su participación mediante un documento de consentimiento informado, en el cual nos comprometíamos a mantener la confidencialidad y anonimato. En este sentido, aclaramos particularmente que ni los profesionales que trabajan en los servicios penitenciarios, ni el juez de vigilancia penitenciaria tendrían acceso a cualquier tipo de información que los 
internos divulgasen durante las sesiones. Igualmente, nos aseguramos de que los internos entendiesen que su participación en el programa era voluntaria, así como que con la misma no podrían alcanzar ningún beneficio y que, en el caso de que decidiesen no participar en el programa, no serían penalizados.

Tabla 1.

Objetivos y contenidos de las sesiones

\begin{tabular}{l}
\hline Sesiones \\
\hline 0. Presentación y \\
evaluación de \\
necesidades de \\
intervención \\
1. La salud mental y \\
sus determinantes
\end{tabular}

2. Interpretación, expresión y control de las emociones

\section{Técnicas de} relajación; Dificultades para dormir y estrategias

4. Distorsión de pensamientos y la importancia de mantener una actitud positiva

5. Autoestima

6. Enf. infecciosas

7. Comunicación con profesionales sanitarios: comunicación asertiva

8. Salud bucodental; Salud en libertad

\section{Objetivos}

Presentación; Intenciones del programa y evaluación de las expectativas y necesidades de los participantes;

Definir la salud mental desde una perspectiva integral; Reflexionar sobre la promoción de la salud mental

Desarrollar habilidades para identificar, reconocer y expresar emociones; Entrenar la respiración abdominal

Aprender técnicas de relajación; Identificar problemas de sueño $\mathrm{y}$ desarrollar estrategias básicas para mejorar la calidad del mismo.

Reflexionar sobre la relación entre pensamientos, emociones y las conductas; Aprender a controlar los pensamientos que distorsionan la interpretación de la realidad.

Desarrollar pensamientos positivos sobre uno mismo.

Evaluar los conocimientos de los participantes sobre las enfermedades infecciosas $y$ desmitificar creencias sobre las mismas

Desarrollar habilidades de comunicación asertiva; Comprender la importancia de una comunicación asertiva en contexto sanitario.

Aprender medidas básicas de higiene oral y prevención de problemas bucodentales; Reflexionar sobre la salud en libertad. Evaluación y cierre del programa

Las sesiones fueron orientadas a la promoción de la educación por pares, uno de los principales objetivos del programa, para lo que se utilizaron estrategias como ejemplificar cómo los contenidos de las diferentes sesiones podían ser aplicados y difundidos por los participantes en la comunidad, reforzando la expresión de otros ejemplos que fueron compartidos de forma espontánea. Cada una de las sesiones terminaba de manera informal, con un pequeño refrigerio, que era aprovechado para obtener una cierta retroalimentación sobre la sesión. Sentimos que este fue un momento fundamental, una vez que durante las sesiones frecuentemente afloraban cuestiones emocionalmente intensas. Este momento constituía una oportunidad para volver al "aquí y ahora", antes de que los internos se retirasen a sus actividades habituales en el centro o a sus celdas.

Para reforzar la difusión de los contenidos de las sesiones, tal y como sugiere García et al., (2004), construimos con los participantes materiales con información sobre salud que fueron colocados en las inmediaciones de la escuela durante un período de aproximadamente tres semanas. Aunque era posible ayudar a los participantes a preparar una presentación oral, la posibilidad de que este momento generase ansiedad a los internos, hizo que considerásemos otra alternativa. Una vez que, con la finalización de programa, no podríamos garantizar condiciones para ayudar a los internos a gestionar su estado emocional, decidimos elaborar materiales escritos para evitar así una excesiva exposición que pudiese dejar a los internos en un estado de fragilidad. Así, consideramos esta opción, ya que entendimos que debemos involucrar a los participantes en las diferentes etapas de cualquiera de estas iniciativas $\mathrm{y}$ todas las decisiones. Los carteles fueron preparados durante algunas sesiones que fueron programadas después de la finalización del programa y que se centraron específicamente en la definición de los métodos más adecuados para la transmisión de mensajes de salud y para la planificación y construcción de los carteles. Como producto final de estas sesiones surgieron cinco carteles dedicados a los siguientes temas: ansiedad y estrés, comunicación asertiva, salud bucodental, problemas para dormir y emociones.

\section{Sobre la evaluación del programa}

La evaluación de los programas proporciona información valiosa para entender los mecanismos por los cuales se produjeron los cambios observados, así como para determinar si las actividades desarrolladas han sido eficaces. En contexto penitenciario no siempre es fácil hacer frente a las numerosas limitaciones institucionales, mantener el enfoque y ajustar los indicadores de éxito a contextos y poblaciones específicas. En este sentido, la evaluación del programa fue condicionada por algunos factores.

La asistencia a las sesiones fue un tema muy sensible. Cuando preparábamos la entrada del equipo en el terreno, algunos profesionales que trabajan en este centro mencionaron la dificultad de mantener la asistencia de los internos em diversas actividades, incluso en el caso de iniciativas pagadas. Por eso, el equipo era consciente de que la asistencia podría ser escasa, y hasta de la posibilidad de tener que cancelar la aplicación del programa por falta de interés de los internos. Conseguimos aplicar la totalidad de las sesiones, contando con la presencia de, en media, seis participantes por sesión. Aunque este resultado, a primera vista, no parece muy positivo, las cuestiones relacionadas con la asistencia a las intervenciones aplicadas en este tipo de contextos requieren una reflexión más profunda.

Es probable que la localización del centro penitenciario haya podido tener alguna influencia en la asistencia a las 
sesiones. Los centros penitenciarios urbanos son frecuentados por estudiantes de grado que pretenden desarrollar sus trabajos de investigación en este contexto, por lo que los internos tienen mayores probabilidades de ser solicitados, varias veces inclusivamente, para participar en estudios o programas. Este fenómeno tiene el efecto de desgastar a los internos, dejándolos menos motivados para su participación. Además, podemos identificar algunos factores relacionados con el funcionamiento de este tipo de instituciones que no facilitan la participación (por ejemplo, la aplicación de sanciones que impiden la asistencia a programas o las huelgas del personal de vigilancia, que obligaba al cambio del horario de visitas, coincidiendo muchas veces con las sesiones semanales). Por otro lado, la elevada proporción de internos que cumplían condenas cortas (36 años), dificultó la adhesión a esta iniciativa debido a la necesidad de tratar cuestiones legales en los momentos en que se desarrollaban las sesiones. Por todos estos motivos, tratándose de un grupo tan pequeño, y conociendo las dificultades que normalmente los profesionales identifican en relación a la asistencia, consideramos que la adhesión fue buena, aunque siempre mejorable, y consideramos que fue un éxito conseguir desarrollar el programa hasta su fin.

El limitado número inicial y final de participantes dificultó realizar una evaluación de resultados (Wynn, Dutta, \& Nelson, 2005). Por este motivo, optamos por realizar una monitorización del proceso, informalmente, durante los minutos finales de cada sesión, así como una evaluación del proceso, en la última sesión a través de un breve cuestionario, cuestionando la adecuación de la implementación del programa desde la perspectiva de la satisfacción de los participantes. A pesar del limitado número de asistentes a la última sesión, decidimos recoger estas opiniones por su valor cualitativo y simbólico. No fueron incluidos los restantes participantes ya que, como se describió anteriormente, muchos de ellos no pudieron participar en todas las sesiones, por varias razones. La evaluación consistía en un cuestionario breve utilizando una escala tipo Likert, incluyendo la comprensión e interés de los contenidos transmitidos, la duración de las sesiones y la aplicación práctica de los contenidos trabajados. También se incluyó una evaluación sobre el equipo de trabajo, más concretamente sobre su conocimientos sobre los temas tratados, su capacidad para transmitir información, y las competencias de comunicación. Las respuestas podían ser completadas a través de respuestas abiertas, con el objetivo de recoger información sobre aquellos aspectos que les habían gustado y aquellos que podrían ser mejorados. De forma general, el programa fue evaluado de forma positiva por los participantes, considerando que los contenidos de las sesiones eran interesantes y prácticos, habiéndose utilizado el tiempo adecuado en cada una de sus abordajes. Igualmente consideraron que el equipo de trabajo dominaba los temas que se trataron consiguiendo transmitir conocimientos de forma adecuada y dando atención al grupo.

Por otro lado, para evaluar el impacto de los carteles en los internos que asistían a la escuela, construimos un breve cuestionario que fue administrado por el equipo de trabajo solicitando información sobre cuestiones relacionadas con los problemas de salud (ej., la importancia que se da a la información sanitaria), así como sobre los carteles y la información que se transmitía (ej. recordar haber visto o leído los carteles; interés, credibilidad, relevancia y utilidad de los carteles para sí mismo o para otros; otras posibles formas de difusión de mensajes de salud).

Respondieron al cuestionario 34 internos. En general, los carteles fueron evaluados muy positivamente, considerándose el contenido atractivo e interesante. La mayoría de los internos $(n=25)$ afirmaron que habían leído los carteles, a pesar de que sólo 22 internos afirmaron recordar haber visto carteles. Esta ligera falta de coherencia en las respuestas puede sugerir, como afirma la literatura, alguna dificultad por parte de los internos para comprender las preguntas a través de un instrumento como un cuestionario. A pesar de que conocíamos esta dificultad, decidimos que no era adecuado proporcionar asistencia a los internos durante el período de respuesta por los problemas éticos que esto suscitaría, ya que se trataba de un proceso en el que se pretendía evaluar nuestro propio trabajo. Sin embargo, a pesar de las posibles inconsistencias en las respuestas, somos de la opinión de que siempre será preferible a conocer la opinión de los destinatarios, aun con sus incoherencias.

La gran mayoría de las respuestas de los internos al cuestionario se agrupan en los dos puntos superiores de la escala ("Muchísimo" y "mucho"). De esta manera, la mayoría de los internos (91\%) atribuye "muchísima" y "mucha" importancia la información divulgada a través de los carteles. Esta tendencia se repite cuando se pregunta a los internos por el interés de la información ( $n=10$ "muchísima" y 15 "mucha"), credibilidad de la misma ( $n=9$ "muchísima" y $n=10$ "mucha"), pertinencia (12 "muchísima" y $n=16$ "mucha") e utilidad de la información sobre salud transmitida por los carteles ( $n=12$ "muchísima" y $n=11$ "mucha"). Esta tendencia de respuesta sugiere que la elección de los temas fue adecuada, sobresaliendo así la importancia de la participación de los internos para despertar el interés y la motivación de los mismos. En otras palabras, parece que la participación de los destinatarios en la intervención es una excelente estrategia para incluir temas que sean del interés de los mismos. Las respuestas al cuestionario también mostraron que una grande parte de los internos $(70.5 \%)$ manifestó su intención de seguir la información incluida en los carteles; transmitir a otros esa información; o divulgar la misma a personas de su círculo social. Para finalizar, debemos destacar el interés que los reclusos que asisten a la escuela mostraron en relación a la recepción de información sobre salud. Apenas una minoría ( $n=1$ interno) respondió que no tendría interés en recibir este tipo de información. De esta forma, podemos reforzar las necesidades de intervención identificadas durante la primera sesión de la intervención.

Somos conscientes que el hecho de haber escogido las inmediaciones de la escuela como zona en la que colocar los carteles limita el impacto de la intervención, ya que posiblemente esto condiciona el grupo diana, una vez que posiblemente la información llegue a un grupo específico 
de internos, posiblemente aquellos más motivados para aprender. Sin embargo, esta decisión se basó, por un lado, en las instrucciones que nos fueron dadas por la institución, y por otro, en una forma de asegurar que los carteles no fueran destruidos y se mantuviesen visibles para la comunidad escolar. Además, consideramos que era en las inmediaciones de la escuela donde solía concentrarse un número considerable de internos, lo que podría asegurar la lectura de los carteles, facilitando así la posibilidad de conseguir cambios de comportamiento en el grupo destinatario.

\section{Conclusión}

Nuestra experiencia en este programa refuerza la idea, defendida por la OMS, de que las prisiones pueden y deben ser contextos de promoción de la salud (Møller et al., 2007). A pesar de ser un contexto adverso, las cárceles también pueden ser consideradas como un entorno estratégico donde las personas pueden ser apoyadas para poder reaccionar mejor a condiciones adversas y fomentar activos para ganar salud (Bustamante et al., 2013). Así, parece fundamental relacionar el contexto físico y social del ambiente penitenciario con el propósito de resocialización que deben tener las penas de prisión. Las prisiones deben proporcionar a los reclusos los medios necesarios para su verdadera reintegración (Zapico, 2009). Como menciona Woodall (2016), las intervenciones suelen prestar más atención al sistema de justicia penal que a la población penitenciaria. Yendo un poco más lejos, la OMS sugiere no limitarse a intentar mejorar la salud de las personas, sino también el espacio físico y social penitenciarios. Aunque la OMS defiende esta idea desde hace unas décadas, la aplicación de este tipo de políticas ha sido excesivamente discreta. Aunque conocemos las limitaciones y dificultades de desarrollar programas de promoción de la salud en este contexto, parece necesario hacer algo más para conseguir un sistema de justicia penal más sano, disminuyendo la inconsistencia entre la teoría y la práctica. Las intervenciones en contexto penitenciario podrán ayudar a atender las necesidades de aquellos sectores de la población más vulnerables y excluidos, pero la promoción de la salud debería empezar mucho antes. De hecho, consideramos que el discurso de la cárcel promotora de salud debería extenderse, no sólo hacia un sistema de justicia penal más sano, sino hacia una sociedad más promotora de salud.

\section{Referencias}

Bustamante, R., Paredes-Carbonell, J. J., Aviñó, D., González, J., Pitrach, C., Martínez, L., \& Arroyo, J. M. (2013). Diseño participativo de una guía para la promoción de la salud mental en el medio penitenciario. Revista Española de Sanidad Penitenciaria, 15, 44-53. Retrieved from: http://scielo.isciii.es/pdf/sanipe/v15n2/02_original1.p df

Coll, C., \& Solé, I. (1989). Aprendizaje significativo y ayuda pedagógica. Cuadernos de Pedagogia, 168, 1620.
Collica-Cox, K. (2014). Counting down. International Journal of Offender Therapy and Comparative Criminology, 58(8), 931-952. doi: 10.1177/0306624X13490660

Comfort, M. L., \& Grinstead, O. (2004). The carceral limb of the public body: Jail inmates, prisoners, and infectious disease. Journal of the International Association of Physicians in AIDS Care, 3(2), 45-48. doi: 10.1177/154510970400300202

Enggist, S., Møller, L., Galea, G., \& Udesen, C. (Eds.) (2014). Prisons and health. Copenhagen, Denmark: World Health Organization. Retrieved from: http://www.euro.who.int/_data/assets/pdf_file/0005/2 49188/Prisons-and-Health.pdf

Equip Vincles Salut (2011). Guía para la promoción de la salud mental en el medio penitenciario. Madrid: Ministerio del Interior y Secretaria General de Instituciones Penitenciarias. Retrieved from: http:/www.institucionpenitenciaria.es/web/export/site s/default/datos/descargables/saludpublica/Guia_Promo cion_Salud_Mental.pdf

García, G., Gutiérrez, E., \& Morante, L. (2004). Bola de nieve. Guía para la formación de usuarios de drogas como agentes de salud. Madrid: Grupo CID. REtrieved from:

http://www.fundacioncsz.org/ArchivosPublicaciones/1 56.pdf

Møller, L., Stöver, H., Jürgens, R., Gathere, A., \& Nikogosian, H. (Eds.) (2007). Health in prisons. A WHO guide to the essentials in prison health. doi: 10.1016/S0140-6736(01)00242-2

Santora, L., Espnes, G. A., \& Lillefjell, M. (2014). Health promotion and prison settings. International Journal of Prisoner Health, 10(1), 27-37. doi: 10.1108/IJPH-082013-0036

Scientific Institute of the Medical Association of German Doctors. (2013). Health promotion for young prisoners: A toolkit for prison staff. Retrieved from: http://ec.europa.eu/chafea/projects/database/fileref/20 091212_d06-01_en_ps.pdf

Watson, $\bar{R}$., Stimpson, A., \& Hostick, T. (2004). Prison health care: A review of the literature. International Journal of Nursing Studies, 41(2), 119-128. http://doi.org/10.1016/S0020-7489(03)00128-7

WHO (1995). Health in prisons Health promotion in the prison setting. Retrieved from: http://apps.who.int/iris/bitstream/10665/107506/1/EU R_ICP_ADA_043\%28S\%29.pdf

Woodall, J. R. (2016). A critical examination of the health promoting prison two decades on. Critical Public Health, 26(5), 615-621. doi: 10.1080/09581596.2016.1156649

Wynn, B. O., Dutta, A., \& Nelson, M. I. (2005). Challenges in program evaluation of health interventions in developing countries. Santa Monica, CA: RAND Corporation. doi: 10.7249/mg402hlth

Zapico, M. (2009). ¿Un derecho fundamental a la reinserción social? Reflexiones acerca del artículo 25.2 de la CE. Anuario da Facultade de Dereito da Universidade da Coruña, 13, 919-944. Retrieved from: http://ruc.udc.es/dspace/bitstream/2183/7505/1/AD_1 3_art_41.pdf 


\section{Agradecimientos}

Este trabajo fue realizado en el ámbito del proyecto "Saúde em cadeia: programa de promoção da literacia em saúde entre pares em contexto penitenciário" (P-131849), financiado por la Fundação Calouste Gulbenkian (FCG). 